\title{
Ensaio clínico duplo-cego randomizado e placebo- controlado com naltrexona e intervenção breve no tratamento ambulatorial da dependência de álcool
}

\author{
A double blind, randomized and placebo-controlled \\ clinical trial with naltrexone and brief intervention in \\ outpatient treatment of alcohol dependence
}

Luís André Castro', Ronaldo Laranjeira ${ }^{2}$

\section{RESUMO}

Objetivo: $O$ objetivo deste estudo é avaliar a eficácia da naltrexona com intervenção breve em pacientes com dependência de álcool. Método: Este estudo é um ensaio clínico randomizado, duplo-cego, placebo-controlado de 12 semanas. A amostra de 71 pacientes foi dividida randomicamente em dois grupos (um recebendo naltrexona e outro placebo). Sujeitos dependentes de álcool foram tratados com 50 mg de naltrexona ou placebo diariamente por 12 semanas. Ambos os grupos de tratamento receberam intervenção breve. Os desfechos clínicos primários para este estudo foram taxa de recaída e mudança no padrão de consumo de álcool. Resultados: Na intenção de tratar, menor porcentagem de sujeitos tratados com naltrexona recaíram (3\% 21\%; $p=0,054)$. Naltrexona com intervenção breve não foi superior ao placebo para diminuir os dias de consumo $(6,2+10,63,05+7,3$; $p=0,478)$, os dias de consumo moderado $(02,2+6,9 ; p=0,345)$ e os dias de consumo pesado $(0,03+0,20,3+0,9 ; p=0,887)$. Naltrexona foi bem tolerada. Os efeitos adversos mais frequentes na presente amostra foram: cefaleia $(25,4 \%)$, sonolência $(20,9 \%)$, náuseas $(16,4 \%)$, hiperfagia (16,4\%), anorexia (14,9\%), ansiedade $(10,4 \%)$, pirose $(10,4 \%)$ e irritabilidade $(10,4 \%)$. Conclusões: Embora o grupo naltrexona tenha demonstrado tendência para reduzir taxa

\section{Palavras-chave}

Naltrexona, alcoolismo, farmacoterapia. de recaída (> 5 doses/dia), não foi encontrada nenhuma diferença em outras variáveis de consumo de álcool entre os grupos naltrexona e placebo. Estudos futuros devem examinar a eficácia desse tipo de combinação de tratamento nos cuidados primários de saúde.

\section{ABSTRACT}

Objective: The objective of this study is to evaluate the efficacy of naltrexone with brief intervention among patients with alcohol dependence. Method: This study is a 12-week randomized, double blind, placebo-controlled clinical trial. The sample of 71 patients was randomly divided in two groups (one receiving naltrexone and the other placebo). Alcohol-dependent

1 Universidade Federal de São Paulo, Escola Paulista de Medicina (Unifesp/EPM), Departamento de Psiquiatria. 2 Universidade Federal de São Paulo, Escola Paulista de Medicina (Unifesp/EPM), Unidade de Pesquisa em Álcool e Drogas (Uniad), Departamento de Psiquiatria.

Endereço para correspondência: Luís André Castro Rua Martins Pena, 236, ap. 64, Tatuapé - 03066-000 - São Paulo, SP Telefone: (11) 9931-8395

E-mail: piancoj@ig.com.br 


\section{Keywords}

Naltrexone, alcoholism, drug therapy. subjects were treated with $50 \mathrm{mg}$ of naltrexone or placebo daily for 12 weeks. Both treatment groups received brief intervention. The primary results for this study were relapse rate and change in drinking behaviors. Results: In the intention-to-treat fewer naltrexone treated subjects relapsed (3\% 21\%; $p=0.054$ ). Naltrexone with brief intervention was not effective in decreasing drinking days $(6.2 \pm 10.63 .05 \pm 7.3 ; p=0.478)$, moderate drinking days $(02.2 \pm 6.9$; $p=0.345)$ and heavy drinking days (0.03 $\pm 0.20 .3 \pm 0.9 ; p=0.887)$. Naltrexone was well tolerated. The most frequent adverse effects in our sample were: headache (25.4\%), drowsiness (20.9\%), nausea (16.4\%), hyperphagia (16.4\%), anorexia (14.9\%), anxiety (10.4\%), heartburn (10.4\%) and irritability (10.4\%). Conclusions: Although the naltrexone group showed a tendency to reduce relapse rate ( $>5$ drinks/day), no differences were found in other alcohol consumption variables between naltrexone and placebo groups. Further studies should examine the efficacy of this kind of treatment combination in the primary health care.

\section{INTRODUÇÃO}

No Brasil, os transtornos relacionados ao uso de álcool são um dos principais problemas de saúde pública. Segundo o I Levantamento Domiciliar sobre o Uso de Drogas Psicotrópicas no Brasil, 11,2\% dos brasileiros que vivem nas 107 maiores cidades do País são dependentes de álcool, o que equivale a 5,2 milhões de pessoas'. No ano de 2000, o dano global à saúde provocado pelo consumo de álcool foi responsável por $4 \%$ de toda a morbidade e mortalidade no planeta 2 .

É sabido que os indivíduos que não preenchem critérios diagnósticos para dependência de álcool são mais resistentes às intervenções psicossociais que focalizam a abstinência como meta de tratamento. Em tais casos, as intervenções breves (IB) demonstraram ser eficazes para reduzir o consumo de álcool, embora o tamanho do efeito do tratamento (ou ) dessas intervenções seja considerado pequeno (inferior a 0,2). Supõe-se que o uso de medicações - entre as quais a naltrexona - possa potencializar os efeitos terapêuticos das $\mathrm{IB}^{3,4}$. A naltrexona é uma intervenção farmacológica comprovadamente eficaz no tratamento da dependência de álcool, pois promove redução das taxas de recaídas e da quantidade e frequência do consumo de álcoo| ${ }^{5-7}$.

Este ensaio clínico foi conduzido com os seguintes objetivos primários:

a) avaliar a eficácia clínica da naltrexona associada à intervenção breve, levando-se em consideração as variáveis dependentes que monitoram o número de dias de consumo leve (até 4 doses/dia), moderado (5 a 9 doses/dia) e pesado (10 ou mais doses/dia) nos últimos 30 dias, bem como as taxas de recaída (ingestão de 5 ou mais doses por dia);

b) estimar a tolerabilidade da naltrexona numa amostra de pacientes ambulatoriais dependentes de álcool, por meio da análise das taxas dos efeitos adversos mais comuns (acima de 10\%).

\section{MÉTODO}

\section{Desenho do estudo}

Trata-se de um ensaio clínico duplo-cego, randomizado, placebo-controlado, de 12 semanas, com amostra de 71 pacientes, constituída por dependentes de álcool que procuraram tratamento ambulatorial na Unidade de Pesquisa em Álcool e Drogas (Uniad-Unifesp) no período de $1^{\circ}$ de agosto de 2001 a 13 de outubro de 2003.

\section{Critérios de admissão}

Os critérios de inclusão para este estudo foram: pacientes de ambos os sexos que preencheram os critérios diagnósticos do Diagnostic and Statistical Manual of Mental Disorders, fourth edition (DSM-IV), 1994, para dependência do álcool, com faixa etária entre 18 e 65 anos de idade, período de abstinência entre 5 e 30 dias, endereço residencial fixo na cidade de São Paulo e que preencheram o consentimento livre e esclarecido padronizado.

Os critérios de exclusão dos pacientes foram: presença de transtornos mentais que demandem internações psiquiátricas ou intervenções farmacológicas para estabilização do quadro psicopatológico, doenças clínicas que contraindiquem a prescrição da naltrexona, transaminases hepáticas com valores três vezes acima do normal, uso de opioides, metadona, cocaína, cannabis e anfetaminas nos últimos 30 dias e uso prévio de dissulfiram, naltrexona e acamprosato.

\section{Procedimentos}

A avaliação inicial constituiu no preenchimento de ficha de identificação, inventário sociodemográico, ficha médica padronizada, para sintomas físicos, questionário sobre consumo de álcool nos últimos 3 meses e 30 dias, respectivamente, consentimento informado e padronizado por escrito, além da realização da entrevista clínica estruturada para 
dependência de álcool e de exames laboratoriais (TGO, TGP, Y-GT, e hemograma completo). Após 5 dias de abstinência, os pacientes admitidos no ensaio clínico foram randomizados, por meio de uma lista de números aleatórios para o grupo naltrexona ( $n=35)$ e o grupo placebo $(n=36)$.

Nas avaliações de seguimento e final foram aplicados os questionários sobre consumo de álcool nos últimos 30 dias e o para monitorar os efeitos adversos da naltrexona, por uma psiquiatra e uma enfermeira. Os pacientes foram orientados a fazer uso da naltrexona durante a recaída. Aqueles que evoluíram com CIWA > 8 receberam tratamento com benzodiazepínicos pelo período de duas semanas até a remissão dos sintomas da síndrome de abstinência alcoólica.

\section{COMITÊ DE ÉTICA EM PESQUISA}

O estudo foi aprovado pelo comitê de ética em pesquisa do Hospital São Paulo (Processo no 046/00).

\section{Análise estatística}

\section{Cálculo amostral}

Para o cálculo do tamanho da amostra, levou-se em consideração a diferença entre os tratamentos (22\%), $a=0,05$ e 1 - $\beta=0,90$. Portanto, o tamanho da amostra proposto para este ensaio clínico é de 129 sujeitos.

\section{Análise estatística inferencial}

$\mathrm{Na}$ análise descritiva das variáveis contínuas, levou-se em consideração a medida de tendência central (média e desvio-padrão). Frequências absolutas e relativas foram calculadas para as variáveis categoriais. Em relação aos testes estatísticos para as variáveis contínuas, foram utilizados o teste $\mathrm{t}$ de Student e a análise dos dados categoriais feita por meio do teste do qui-quadrado. Para comparar as variáveis de interesse pré e pós-tratamento e os dois tipos de tratamento (naltrexona e placebo), foram feitas análises de variância com medidas repetidas, realizadas com 33 pacientes em cada tratamento. Para a análise das variáveis categoriais, foi empregado o teste do qui-quadrado ou o teste exato de Fisher, quando a amostra era menor. Para todas as análises o nível de significância escolhido foi de 5\% (ou $\mathrm{P}<0,05$, bicaudal). Foram excluídos da análise inferencial os pacientes que compareceram a apenas uma sessão da IB.

\section{RESULTADOS}

\section{Caracterização da amostra}

Neste estudo não foram encontradas diferenças entre os grupos, em relação a sexo $\left(x^{2}=0,747, p=0,387\right)$, idade $(t=0,533, p=0,596)$, estado civil $\left(x^{2}=3,315, p=0,069\right)$ e situação empregatícia ( $\left.x^{2}=0,676, p=0,411\right)$. Quanto à gravidade da dependência, ambos os grupos também não diferiram em termos de escores do SADD $(t=-1,249, p=0,215)$ e da escala OCDS $(t=-0,323, p=0,748)$.

\section{Padrão de consumo de álcool}

Ao final do ensaio clínico não foram encontradas diferenças estatisticamente significativas entre os grupos, quanto ao número de dias de consumo $(6,2 \pm 10,63,05 \pm 7,3$; $t=162,815 ; p=0,478)$, dias de consumo moderado $(02,2 \pm$ $6,9 ; t=0,906 ; p=0,345)$ e dias de consumo pesado $(0,03 \pm$ $0,20,3 \pm 0,9 ; t=-3,403 ; p=0,887)$. Contudo, o grupo placebo apresentou uma redução dos dias de consumo leve estatisticamente significativa $(4,33 \pm 8,81,0 \pm 3,3, t=7,445$; $p=0,008)$.

\section{Taxas de recaídas}

Os pacientes tratados com naltrexona evoluíram com tendência para taxas inferiores de recaídas em relação ao grupo placebo (3\% 21\%, teste exato de Fisher; $p=0,054)$. Foram excluídos cinco pacientes dessa análise, pois eles abandonaram o estudo antes da segunda sessão de intervenção breve, impossibilitando a avaliação por intenção de tratar.

\section{Efeitos adversos}

Neste ensaio clínico não foram encontradas diferenças estatisticamente significativas entre os grupos para nenhum dos efeitos adversos investigados: cefaleia $\left(x^{2}=0,008\right.$; $p=0,931)$, sonolência $\left(x^{2}=0,836 ; p=0,361\right)$, hiperfagia $\left(x^{2}=1,310\right.$; $p=0,252)$, náuseas $\left(x^{2}=0,769 ; p=0,380\right)$, anorexia $\left(x^{2}=0,509\right.$; $p=0,475)$, ansiedade $\left(x^{2}=5,775 ; p=0,152\right)$, azia $\left(x^{2}=2,868\right.$; $p=0,090)$ e irritabilidade $\left(x^{2}=0,330 ; p=0,566\right)$. Foram relacionados os efeitos adversos mais comuns (acima de 10\%) entre os pacientes de ambos os grupos, que frequentaram pelo menos duas sessões de intervenção breve $(n=67)$.

\section{DISCUSSÃO}

Este é o primeiro ensaio clínico randomizado (ECR), duplocego e placebo-controlado conduzido no Brasil com o objetivo de avaliar a eficácia clínica e a tolerabilidade da naltrexona no tratamento ambulatorial da dependência do álcool na população em questão. Os resultados apresentados são preliminares, em decorrência de limitações metodológicas discutidas posteriormente. Além disso, na própria literatura existem poucos artigos que abordam a efetividade de intervenções farmacológicas vinculadas à IB na rede primária de saúde. A IB é uma intervenção psicossocial bastante investigada no manejo de indivíduos com abuso de álcool que não preenchem critérios diagnósticos para dependência de álcool. 
Na amostra em questão, os sujeitos submetidos a placebo apresentaram redução estatisticamente significativa dos dias de consumo leve nos últimos 30 dias $(p=0,008)$, quando comparados ao grupo tratado com naltrexona. É sabido que os antagonistas opioides são eficazes na redução do consumo pesado de álcool (acima de 5 doses por dia), tendo menor impacto no consumo de menor gravidade. Além disso, não se pode descartar o efeito placebo neste estudo. As taxas de resposta ao placebo são elevadas na psiquiatria (65\% na depressão maior e entre $20 \%$ a $50 \%$ na esquizofrenia). Existem evidências científicas para se considerar a dopamina e as endorfinas como os principais mediadores bioquímicos da resposta placebo. Portanto, a diferença estatisticamente significativa a favor do placebo nos indivíduos que ingeriram álcool de forma leve nos últimos 30 dias pode ser atribuída ao deficiente bloqueio dos receptores opioides pela naltrexona, pelo menos com a dose de $50 \mathrm{mg} / \mathrm{dia}^{8,9}$.

Em relação aos dias de consumo $(p=0,478)$, dias de consumo moderado $(p=0,345)$ e dias de consumo pesado $(p=0,887)$, não foram detectadas diferenças entre os grupos. Na metanálise conduzida por Srisurapanont e Jarusuraisin? também não foram encontradas diferenças estatisticamente significativas entre naltrexona e placebo em relação aos dias de consumo (WMD $=-1,96$ IC 95\% -5,47 a 1,56). Segundo Rubio et al.10, isso pode ser explicado pela natureza da IB, que se diferencia de outras intervenções psicossociais (por exemplo, terapia cognitivo-comportamental) que preconizam como meta de tratamento a abstinência. Outra explicação plausível foi a duração da IB. No ensaio clínico controlado conduzido por O'Malley et al."11 não foi encontrada diferença entre a TCC e IB (denominada Primary Care Management - PCM) para diferentes desfechos clínicos antes de 10 semanas. No entanto, essa diferença aflorou na fase de manutenção a favor do grupo tratado com naltrexona associado a IB, levando-se em consideração as taxas de sujeitos que mantiveram os resultados iniciais ao tratamento, definido pelo consumo pesado de álcool pelo período máximo de 2 dias nos últimos 28 dias, ou então, a ingestão de pelo menos $60 \%$ da medicação nas primeiras 10 semanas do estudo. Outras variáveis clínicas que evidenciaram superioridade sobre o placebo incluíram as taxas de dias de abstinência, o número de doses por dia de consumo e os níveis séricos de $\gamma$-GT. Esses achados sugerem que é vantajoso manter um coadjuvante farmacológico à IB por períodos de até 6 meses. Outras hipóteses plausíveis são as características clínicas da amostra oriunda de um ambulatório especializado no tratamento de dependentes de álcool e a intensidade da intervenção psicossocial. Esses sujeitos, quando comparados aos pacientes de outros ambulatórios, são mais graves, conforme os resultados de um estudo longitudinal de Fontes et al.12, que evidenciou incidência maior de problemas de saúde mental entre os pacientes dependentes de álcool de um ambulatório especializado no tra- tamento do alcoolismo, quando comparados aos pacientes de um ambulatório de gastroenterologia. Entre os pacientes do ambulatório especializado predomina a síndrome de dependência grave (61,5\% da amostra). O padrão de consumo de álcool é mais pesado em termos de quantidade e frequência. A taxa de mortalidade é alta, em torno de 19,5\%. Outro achado importante deste estudo é que os pacientes que frequentaram a Uniad eram mais motivados, além de estarem mais propensos a mudanças, haja vista os índices elevados de contemplação, ação e manutenção. Os primeiros três meses de tratamento é um período importante, haja vista as taxas elevadas de recaídas. Quando se intensifica o atendimento nesse período, independentemente da intervenção farmacológica utilizada (placebo ou não), as diferenças entre os grupos são reduzidas. É importante ressaltar que as intervenções breves dos ECR com naltrexona foram mais intensivas em comparação àquelas utilizadas com bebedores pesados na rede primária de saúde. Portanto, a intensidade da intervenção psicossocial pode afetar a eficácia terapêutica da naltrexona.

Outra variável clínica investigada foi a taxa de recaída. Cerca de 3\% dos pacientes tratados com naltrexona evoluíram com recaídas, enquanto no grupo placebo essa porcentagem foi de $21 \%(p=0,054)$. A taxa de recaída do presente estudo foi inferior aos três RCR que a estimaram entre $35 \%$ e $53 \%{ }^{10,13,14}$. Nessa casuística o risco relativo (RR) foi inferior ao encontrado na metanálise de Srisurapanont ${ }^{7}$ $(0,14$ 0,64), ou seja, o risco de recaída é de $14 \%$ no grupo submetido à naltrexona, quando comparado ao grupo placebo. Outra medida de efeito a ser mencionada é a redução do risco relativo (RRR) obtido com a naltrexona, conhecido também como eficácia. Conclui-se que o uso da naltrexona reduziu em $86 \%$ o risco de recaída na amostra em questão. Na metanálise conduzida por Srisurapanont e Jarusuraisin', o RRR foi estimado em 36\%. O número necessário para tratar (NNT) foi calculado em torno de 5. Portanto, previne-se um caso de recaída em cada cinco pacientes com dependência de álcool que fazem uso de naltrexona. Novamente, os valores encontrados foram inferiores aos citados na metanálise de Srisurapanont e Jarusuraisin ${ }^{7}$ $(N N T=7)$. Essa tendência estatística é um indicador de que os antagonistas opioides são efetivos para prevenir recaídas, pois eles promovem atenuação dos efeitos prazerosos associados ao consumo de álcool, por meio do bloqueio dos receptores opioides. O álcool estimula a atividade opioide endógena ao induzir a liberação dos peptídeos endógenos (encefalinas e $\beta$-endorfinas) no sistema nervoso central (SNC), que por sua vez mediam a liberação de dopamina no núcleo accumbens, principal estrutura do sistema de recompensa cerebral envolvida com a percepção prazerosa das drogas psicoativas. Portanto, mesmo que o paciente consuma álcool, a probabilidade de evoluir para uma recaída é menor, em decorrência do efeito da naltrexona. 
A adesão é uma variável considerada clinicamente importante no tratamento da dependência de álcool e de outras drogas. Segundo Marques e Furtado ${ }^{15}$, as taxas de abandono são mais frequentes na fase de manutenção, próximo da alta, sendo estimada em 30\%. Dessa forma, no tratamento da dependência de álcool devem ser envolvidas intervenções psicossociais e farmacológicas, que muitas vezes, ao atuarem de forma sinérgica, possibilitam aumentar a adesão ao tratamento. Portanto, oferecer estratégias comportamentais ou farmacológicas na fase inicial do tratamento pode diminuir as taxas de abandono.

Neste ensaio clínico, a adesão ao tratamento foi similar entre o grupo placebo e os sujeitos que foram tratados com naltrexona (83\% 71\%; $p=0,230)$. O número de sessões frequentadas ao final do estudo também confirmou essa tendência $(3,8 \pm 1,43,9 \pm 1,3 ; p=0,783)$. Esses achados são semelhantes aos resultados de O'Malley et al."', que também não encontraram diferenças em relação à adesão ao tratamento entre naltrexona e placebo (semanas em tratamento; $18,1 \pm 8,8$ 16,1 $\pm 9,2 ; p=0,41$ ). Segundo Srisurapanont e Jarusuraisin ${ }^{7}$, as taxas de descontinuação nas primeiras 12 semanas de tratamento são similares entre os grupos. Entretanto, as taxas de no grupo tratado com naltrexona são consideradas elevadas (36\% com $\mathrm{RR}=0,85-\mathrm{IC} 95 \%=0,72-1,01)$ e podem comprometer a validade dos resultados. Recomenda-se acrescentar às intervenções psicossociais estratégias que aumentem a aderência ao tratamento. É importante ressaltar que os abandono prévio (antes da primeira consulta) e o precoce (depois da primeira consulta) são considerados piores do ponto de vista do prognóstico, quando comparados à interrupção tardia do tratamento.

$\mathrm{Na}$ amostra do presente estudo, a naltrexona foi bem tolerada pelos pacientes, visto que não foram encontradas diferenças entre o grupo placebo e o grupo naltrexona. Os principais efeitos adversos nesta casuística foram cefaleia $(25,4 \%)$, sonolência $(20,9 \%)$ e náuseas $(16,4 \%)$. Segundo Srisurapanont e Jarusuraisin', os efeitos adversos mais frequentes nos indivíduos tratados com naltrexona são náuseas, tontura e fadiga. Evidencia-se um perfil de efeitos adversos diferentes entre as duas amostras, exceto as náuseas que são consideradas o principal efeito adverso da naltrexona oral, que geralmente coincide com os níveis plasmáticos atingidos num período de até 90 minutos depois da ingestão do medicamento. Em nossa casuística, a naltrexona aumentou em 200\% o risco de náuseas em relação ao grupo placebo. O número necessário de indivíduos para apresentar náuseas como um efeito indesejado da naltrexona foi estimado em 6 (NNH - number needed to harm). A taxa de descontinuação associada aos efeitos adversos é da ordem de 15\%. Segundo Croop ${ }^{16}$, as náuseas são responsáveis por $6 \%$ desses abandonos. Na amostra em questão, não foram observados abandonos causados por reações adversas, apesar da incidência maior na população do presente estudo. Recomenda-se acrescentar às intervenções psicossociais estratégias de manejo clínico dos principais efeitos adversos da naltrexona, tais como a ingestão com alimentos e mudanças nos horários de administração do medicamento.

Os autores concluem que são necessários maiores estudos com intervenções combinadas na rede primária de saúde, a fim de identificar aqueles indivíduos com características clínicas que possam predizer melhor resposta terapêutica às intervenções farmacológicas atualmente disponíveis (dissulfiram, naltrexona e acamprosato). Os resultados iniciais dos ensaios clínicos abertos conduzidos por Bohn ${ }^{17}$, O'Connor et al.13 e Kranzler et al. ${ }^{3}$ não foram replicados na presente amostra. Outro aspecto importante é a diversidade dos critérios de inclusão entre os estudos, que variaram desde indivíduos caracterizados como bebedores-problemas até aqueles com dependência leve de álcool. A principal repercussão dessa observação é que a maioria dos sujeitos envolvidos nos ensaios clínicos abertos apresentava dependência leve ou moderada. A IB empregada nesses estudos pode ser considerada mais intensiva em comparação àquelas utilizadas com pacientes que não preenchem critérios diagnósticos para dependência de álcool, prejudicando qualquer tipo de análise comparativa com outros estudos que investigaram previamente a eficácia da IB. É controverso se as IB para uso nocivo de álcool possam ser replicadas na íntegra com os dependentes de álcool. Entendemos que são necessárias algumas adequações, tais como sessões de seguimento realizadas em intervalos menores, ou seja, aumento da periodicidade dos atendimentos.

\section{Limitações metodológicas}

Entre as principais limitações metodológicas deste ensaio clínico ressalta-se o tamanho de amostra insuficiente para detectar uma diferença entre os grupos (por exemplo, 40\%). Portanto, os resultados não significativos deste ECR podem estar associados à falta de poder estatístico. O poder de um estudo é definido como a capacidade que ele tem para demonstrar uma diferença estatisticamente significante entre o grupo experimental e o grupo-controle, supondo-se que tal efeito de tratamento exista. Os dois maiores contribuintes para o poder de um estudo são o tamanho do efeito (effect size) e o tamanho da amostra. O tamanho do efeito é a diferença entre as taxas de resposta do grupo com medicamento e as do grupo com placebo. Esse efeito pode ser ou não clinicamente relevante, dependendo da população estudada, principalmente se a condição clínica investigada apresentar taxas elevadas de resposta ao placebo. Entretanto, a maioria dos ECR que compuseram a revisão sistemática de Srisurapanont e Jarusuraisin ${ }^{7}$ também apresentou uma amostra considerada pequena. Por exemplo, para um poder de estudo de 80\% e um índice de significância de 0,05, um estudo com dois grupos de intervenções necessita de pelo menos 87 sujeitos em cada grupo para detectar um grau 
intermediário de efeito do tratamento, levando-se em consideração variáveis dicotômicas. Dos 24 ECR, apenas três obtiveram 87 sujeitos em cada grupo. ECR com amostras pequenas (40 sujeitos em cada grupo) predispõem a erros tipo II.

Os resultados dos testes de significância estatística, por meio dos seus níveis de significância (ou valor de P), costumam ser interpretados equivocadamente como medidas de efeito de uma intervenção. O que mede a magnitude do efeito de uma intervenção é a eficácia, a redução absoluta de risco e o número necessário para tratar. Os valores de P apenas informam a probabilidade de que uma associação seja um achado falso-positivo decorrente do acaso (erro tipo I). Um nível de significância baixo ( $p=5 \%)$ não quer dizer que exista uma forte associação, mas apenas que existe uma forte evidência de que o efeito observado não seja decorrente do acaso. É importante ressaltar que o fato de um resultado não ser estatisticamente significativo não deve ser interpretado como evidência de ausência de efeito da intervenção, mas sim de que as evidências contra a hipótese nula são fracas ${ }^{18}$.

Segundo os resultados de O'Malley et al.1" , a duração do EC é um importante preditor de resposta, já que as diferenças entre o grupo placebo e o grupo naltrexona afloraram ao final de 6 meses. A maioria dos ECR com naltrexona tem uma duração considerada curta. Dos 24 ECR que constituíram a revisão sistemática de Srisurapanont e Jarusuraisin?, apenas 8 apresentaram duração acima de 12 semanas.

\section{CONCLUSÕES}

Neste ECR não foram detectadas diferenças estatisticamente significativas entre os grupos em relação aos dias de consumo $(p=0,478)$, dias de consumo moderado $(p=0,345)$ e dias de consumo pesado ( $p=0,887)$. O grupo naltrexona demonstrou tendência estatística não significativa para reduzir as taxas de recaída (acima de 5 doses/dia).

A naltrexona foi bem tolerada pelos pacientes, visto que não foram encontradas diferenças entre o grupo placebo e o grupo naltrexona. Os principais efeitos adversos na presente casuística foram cefaleia (25,4\%), sonolência $(20,9 \%)$ e náuseas (16,4\%). Na amostra em questão não foram observados abandonos causados por reações adversas.

Neste ECR, a adesão ao tratamento foi similar entre o grupo placebo e os sujeitos que foram tratados com naltrexona ( $83 \%$ versus $71 \% ; p=0,230$ ). O número de sessões frequentadas ao final do estudo também confirmou essa tendência $(3,8 \pm 1,43,9 \pm 1,3 ; p=0,783)$.

Cerca de $3 \%$ dos pacientes tratados com naltrexona evoluíram com recaídas, enquanto no grupo placebo essa porcentagem foi de $21 \%(p=0,054)$. O número necessário para tratar (NNT) foi calculado em torno de 5. Portanto, previne-se um caso de recaída a cada cinco pacientes com dependência de álcool que fazem uso de naltrexona.
Os resultados da amostra do presente estudo reforçam a hipótese do uso da naltrexona por períodos acima de seis meses, quando se associa uma IB. Uma das explicações plausíveis é que a intervenção breve se distingue por incluir, entre as suas metas de tratamento, a redução do consumo de álcool, e não somente a abstinência. Além disso, é uma intervenção menos intensiva, quando comparada à TCC. Portanto, preconiza-se que o tratamento de manutenção com naltrexona associado à IB deva ser mantido por um período maior do que 12 semanas.

\section{AGRADECIMENTOS}

Este estudo teve o apoio financeiro da Fundação de Amparo à Pesquisa do Estado de São Paulo (Fapesp) - Processo no 00/03690-8). A condução deste ensaio clínico foi possível em virtude da participação e da colaboração da psiquiatra Alessandra Diehl Reis e da enfermeira Elda de Oliveira.

Manuscrito baseado na tese de doutorado "Ensaio clínico duplo-cego, randomizado e placebo-controlado com naltrexona associado à intervenção breve no tratamento ambulatorial da dependência do álcool", apresentada na Escola Paulista de Medicina da Universidade Federal de São Paulo (EPM/Unifesp), em 2004

Trabalho realizado na Unidade de Pesquisa em Álcool e Drogas (Uniad), EPM/Unifesp.

Estudo subvencionado pela Fapesp.

\section{REFERÊNCIAS}

1. Carlini EA, Galduróz JCF, Noto AR, Nappo SA. I Levantamento Domiciliar sobre o Uso de Drogas Psicotrópicas no Brasil - 2001. Centro Brasileiro de Informações sobre Drogas Psicotrópicas - Departamento de Psicobiologia da Escola Paulista de Medicina e SENAD - Secretaria Nacional Antidrogas, Presidência da República, Gabinete de Segurança Nacional; 2002.

2. Meloni JN, Laranjeira R. Custo social e de saúde do uso do álcool. Rev Bras Psiquiatr. 2004; 26(Suppl I):7-10.

3. Kranzler HR, Tennem H, Penta C, Bohn MJ. Targeted naltrexone treatment of early problem drinkers. Addict Behav. 1997;22(3):431-6.

4. Fiellin DA, Carrington $R, O^{\prime}$ Connor PG. New therapies for alcohol problems: application to primary care. Am J Med. 2000;108:227-37.

5. Streeton C, Whelan G. Naltrexone, a relapse prevention maintenance treatment of alcohol dependence: a meta-analysis of randomized controlled trials. Alcohol Alcohol. 2001;36(6):544-52.

6. Bouza C, Angeles M, Muñoz A, Amate JM. Efficacy and safety of naltrexone and acamprosate in the treatment of alcohol dependence: a systematic review. Addiction. 2004;99:811-28.

7. Srisurapanont M, Jarusuraisin N. Naltrexone for the treatment of alcoholism: a meta-analysis of randomized controlled trials. Opioid. Int J Neuropsychopharmacol. 2005;8(2):267-80.

8. Elkis H, Gattaz WF. Revivendo o antigo sermão da medicina com o efeito placebo. Rev Bras Psiquiatr. 2000;22(4):153-4.

9. Cho HJ. Algumas recomendações para estudos com placebo. Rev Bras Psiquiatr. 2005; 27(4):336-40. 
10. Rubio G, Manzanares J, López-Muñoz F, Álamo C, Ponce G, Jiménez-Arriero MA, et al. Naltrexone improves outcome of a controlled drinking program. J Subst Abuse Treat. 2002;23:361-6.

11. O'Malley SS, Rounsaville BJ, Farren C, Namkoong K, Wu R, Robinson J, et al. Initial and maintenance naltrexona treatment for alcohol dependence using primary care vs specialty care. Arch Intern Med. 2003;163:1695-704.

12. Fontes A, Figlie NB, Laranjeira R. 0 comportamento de beber entre dependentes de álcool: estudo de seguimento. Rev Psiq Clin. 2006;33(6):304-12.

13. $O^{\prime}$ Connor PG, Farren CK, Rounsaville BJ, O'Malley SS. A preliminary investigation of the management of alcohol dependence with naltrexone by primary care providers. Am J Med. 1997;103:477-82.
14. Latt NC, Jurd S, Houseman J, Wutzkes S. Naltrexone in alcohol dependence: a randomized controlled trial of effectiveness in a standard clinical setting. MJA. 2002;176:530-4.

15. Marques ACPR, Furtado EF. Intervenções breves para problemas relacionados ao álcool. Rev Bras Psiquiatr. 2004;26(Suppl 1):28-32.

16. Croop RS, Faulkner EB, Lubriola DF. The safety profile of naltrexone in the treatment of alcoholism. Arch Gen Psychiatry. 1997;54:1130-5.

17. Bohn MJ, Kranzler HR, Beazoglou BS, Staehler BA. Naltrexone and brief counseling to reduce heavy drinking: results of a small clinical trial. Am J Addiction. 1994;3:91-9.

18. Coutinho ESF, Da Cunha GM. Conceitos básicos de epidemiologia e estatística para a leitura de ensaios clínicos controlados. Rev Bras Psiquiatr. 2005;27(02):146-51. 